\title{
Anorthite: thermal equation of state to high pressures
}

\author{
Raymond Jeanloz ${ }^{\star}$ and Thomas J. Ahrens \\ Seismological Laboratory, Division of Geological and Planetary Sciences, \\ California Institute of Technology, Pasadena, California 91125, USA
}

Received 1979 August 29

Summary. We present shock-wave (Hugoniot) data on single-crystal and porous anorthite $\left(\mathrm{CaAl}_{2} \mathrm{Si}_{2} \mathrm{O}_{8}\right)$ to pressures of $120 \mathrm{GPa}$. These data are inverted to yield values of the Grüneisen parameter $(\gamma)$, adiabatic bulk modulus $\left(K_{\mathrm{S}}\right)$ and coefficient of thermal expansion $(\alpha)$ over a broad range of pressures and temperatures which in turn are used to reduce the raw Hugoniot data and construct an experimentally-based, high-pressure thermal equation of state for anorthite. We find surprisingly high values of $\gamma$ which decrease from about 2.2 to 1.2 over the density range 3.4 to 5.0 $\mathrm{Mg} \mathrm{m}^{-3}$. Our data clearly indicate that whereas the zeroth order anharmonic (quasi-harmonic) properties such as $\gamma$ and $\alpha$ decrease upon compression of a single phase, these properties apparently increase dramatically (200 per cent or more) in going from a low to a high pressure phase. The results for anorthite also support the hypothesis that higher-order anharmonic contributions to the thermal properties decrease more rapidly upon compression than the lowest order anharmonicities. We find an initial density $\rho_{0} \sim 3.4 \mathrm{Mg} \mathrm{m}^{-3}$ for the 'high-pressure phase' portion of the Hugoniot, with an initial value of $K_{\mathrm{S}}$ essentially identical to that of anorthite at zero pressure $(90 \mathrm{GPa})$. This is surprising in light of recently documented candidate high-pressure assemblages for anorthite with significantly higher densities, and it raises the question of the non-equilibrium nature of Hugoniot data. By correcting the properties of anorthite to lower mantle conditions we find that although the density of anorthite is comparable to that of the lowermost mantle, its bulk modulus is considerably less, hence making enrichment in the mantle implausible except perhaps near its base.

\section{Introduction}

Anorthite is a mineral of particular geochemical interest because of its refractory nature. Both theoretical and observational evidence suggest that it is among the first high temperature condensates that form from the solar nebula and as such may be an important phase in

\footnotetext{
^ Present address: Department of Geological Sciences, Harvard University, Cambridge, Massachusetts 02138 , USA.
} 
the earliest accretional history of the planets (e.g. Marvin, Wood \& Dickey 1970; Grossman \& Larimer 1974). Indeed, according to inhomogeneous accretional models the present internal zonation of the planets is thought to reflect, at least in a broad sense, the accumulation of first the more refractory condensates followed by successively more volatile-rich material (e.g. Turekian \& Clark 1969; Clark, Turekian \& Grossman 1972). Hence anorthite and other calcium-aluminium minerals may well be enriched deep within the Earth and, if stable against buoyancy forces, may remain there from the earliest times of the Earth's formation. Recent evidence has, in fact, suggested that a significant portion of the Earth's mantle could be quite enriched in a calcium component: the mineral $\mathrm{CaO}$ at high pressures has properties which are virtually indistinguishable from those of much of the lower mantle (Jeanloz et al. 1979). Except for the few hundred kilometres near its base, however, the lower mantle appears to be relatively homogeneous according to seismological observations, and the most plausible location of a chemically distinct zone might be near the bottom of the mantle (Anderson 1975; Jeanloz \& Richter 1979).

The purpose of this paper is to present new high pressure data on anorthite which provide among the most complete high-pressure, high-temperature characterizations of any silicates to date. We have carried out shock-wave experiments both on single-crystal anorthite and on anorthite containing a substantial initial porosity. Because the states achieved in the latter are much hotter than those produced in the non-porous (single-crystal) samples under shock, the temperature-dependence of the high-pressure equation of state can be directly evaluated (e.g. Keeler \& Royce 1971). Thus, we derive a thermal equation of state for anorthite to pressures in excess of $120 \mathrm{GPa}$ directly from our data and with virtually no theoretical constraints on the form of such an equation of state. As a result, we have experimentally based values over a wide range of pressures and temperatures of such thermal properties as the Grüneisen parameter and coefficient of thermal expansion, as well as the pressure- and temperature-dependent bulk modulus. This in turn allows us to make a direct comparison between anorthite at high pressures and the Earth's interior, and leads us to the conclusion that although the density of anorthite is consistent with that of the lower mantle, the bulk modulus probably precludes significant amounts of anorthite being present except, possibly, in the lowermost portion of the mantle ( $D^{\prime \prime}$ region). A modest enrichment of anorthite (which would be stable at the base of the mantle) is allowed by our data, although none is required.

The porous anorthite used in our experiments is a lunar highlands rock (sample 60025). Its response to dynamic compression is of intrinsic interest for better understanding the cratering properties of the lunar surface. Because of its extremely primitive character, the properties of 60025 are of particular interest for modelling the late-stage accretion and early evolution of the Moon. These considerations have been discussed more fully by Jeanloz \& Ahrens (1978), in which some of the data which are described below were first presented.

\section{Experimental}

Euhedral, single-crystals of a 'transitional' anorthite from Miyake-zima, Izu Islands, Japan (cf. Gay 1954; Müller, Wenk \& Thomas 1972; McLaren 1973) were used as non-porous samples; their composition corresponds to $\mathrm{An}_{95.4} \mathrm{Ab}_{4.5} \mathrm{Or}_{0.1}$ (representative analysis in Table 1). Samples were oriented morphologically for shock-wave propagation along the [010] direction and were prepared so as to avoid the small amounts of olivine $\left(\mathrm{Fo}_{83} \mathrm{Fa}_{17}\right)$ inclusions present in the anorthite crystals. The porous anorthite samples were cut from lunar anorthosite 60025.36, .174 as previously described (Jeanloz \& Ahrens 1978). 
Table 1. Electron microprobe analy sis of single-crystal anorthite (Miyake-zima, Japan).

\begin{tabular}{|c|c|}
\hline Oxide & Weight (per cent) \\
\hline $\mathrm{Na}_{2} \mathrm{O}$ & 0.51 \\
\hline $\mathrm{MgO}$ & 0.04 \\
\hline $\mathrm{Al}_{2} \mathrm{O}_{3}$ & 35.28 \\
\hline $\mathrm{SiO}_{2}$ & 44.19 \\
\hline $\mathrm{K}_{2} \mathrm{O}$ & 0.02 \\
\hline $\mathrm{CaO}$ & 19.57 \\
\hline $\mathrm{FeO}$ & 0.66 \\
\hline Total & 100.27 \\
\hline
\end{tabular}

The experimental techniques used in this study have been presented elsewhere and are only briefly discussed here (see Ahrens, Jackson \& Jeanloz 1977; Jeanloz \& Ahrens 1977, 1978, 1980). The initial densities of samples are determined by an Archimedean and a bulk technique for non-porous and porous samples respectively. Properly characterizing the initial densities of the porous samples is particularly important for obtaining data of high quality (Jeanloz \& Ahrens 1978). Shock experiments were carried out using a two-stage, light-gas gun or a $40 \mathrm{~mm}$ bore, propellant gun to accelerate projectiles to velocities between 2.3 and $6.6 \mathrm{~km} \mathrm{~s}^{-1}$. In each experiment, the impact velocity of the projectile is measured, as is the velocity of the shock wave which is generated in the sample upon impact by the projectile. The shock-wave velocity is determined by measuring the travel time of the shock-wave through the sample (of known thickness) by way of a rotating-mirror or an image-converter streak camera; details of the data reduction procedure are given in Jeanloz \& Ahrens (1980). The Hugoniot state is then determined by applying impedance-matching conditions (Hugoniot equations) to the measured initial density, impact velocity and shock-wave velocity (Walsh \& Christian 1955). A partially-or fully-released state is also determined by a free-surface or buffer impedance mismatch measurement, reduced by way of the Riemannintegral formalism (e.g. Rice, McQueen \& Walsh 1958; Lyzenga \& Ahrens 1978). The standard equations of state of McQueen et al. (1970) for W, Ta and $2024 \mathrm{Al}$ alloy, and of Wackerle (1962) and Jackson \& Ahrens (1979) for fused quartz were used in reducing these data.

\section{Results}

The results of the present experiments on single-crystal and porous anorthite are given in Tables 2 and 3, respectively, and are shown in Figs 1 and 2; for convenience, the data from Jeanloz \& Ahrens (1978) for anorthosite 60025 are included, with slight corrections. Despite variations in composition and porosity, the present data can essentially be considered representative of endmember anor thite $\left(\mathrm{CaAl}_{2} \mathrm{Si}_{2} \mathrm{O}_{8}\right)$ with 0 and 19 per cent initial porosity. Although the data of McQueen, Marsh \& Fritz (1967) for Tahawus anorthosite correspond to a more sodic plagioclase $\left(\mathrm{An}_{49}\right)$, these are indistinguishable from the present (non-porous) data because the Tahawus anorthosite contains enough pyroxene to increase its initial density to that of anorthite (Figs 1 and 2). Hence the single-crystal anorthite and Tahawus anorthosite data are reduced together for determining the properties of anorthite at high pressures (see Jeanloz \& Ahrens 1978). 


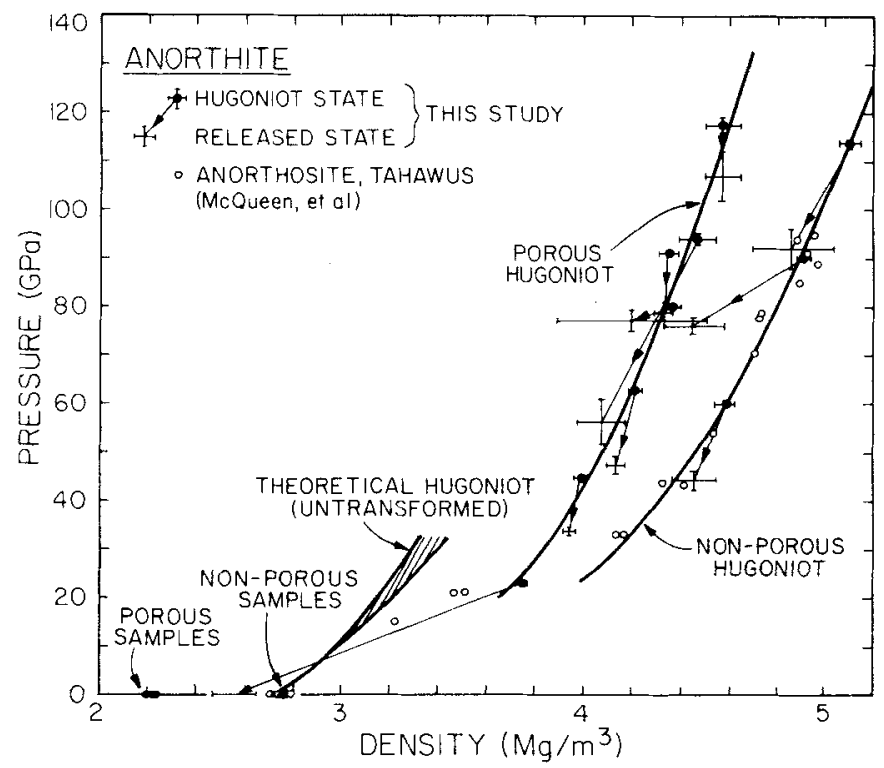

Figure 1. Anorthite and anorthosite shock-wave data from the present study and that of McQueen $e t$ al. (1967) compared with a theoretical Hugoniot for anorthite (its uncertainty corresponds to variations in the assumed pressure derivative of the bulk modulus between 4 and 6 ). The porous and non-porous Hugoniot curves are from the fit listed in Table 4.

All of the present data are in the 'high-pressure phase' regime (e.g. McQueen et al. 1967) and for comparison a theoretical Hugoniot is shown in Figs 1 and 2 for the zero-pressure structure of anorthite. This is constructed (McQueen, Fritz \& Marsh 1963; Davies \& Gaffney 1973) from a third-order Eulerian finite-strain adiabat, constrained by recent ultrasonic data for anorthite (Liebermann \& Ringwood 1976). The Tahawus anorthosite data extrapolate to this theoretical Hugoniot at about $10.3( \pm 3) \mathrm{GPa}$ pressure (Fig. 1), indicating that the

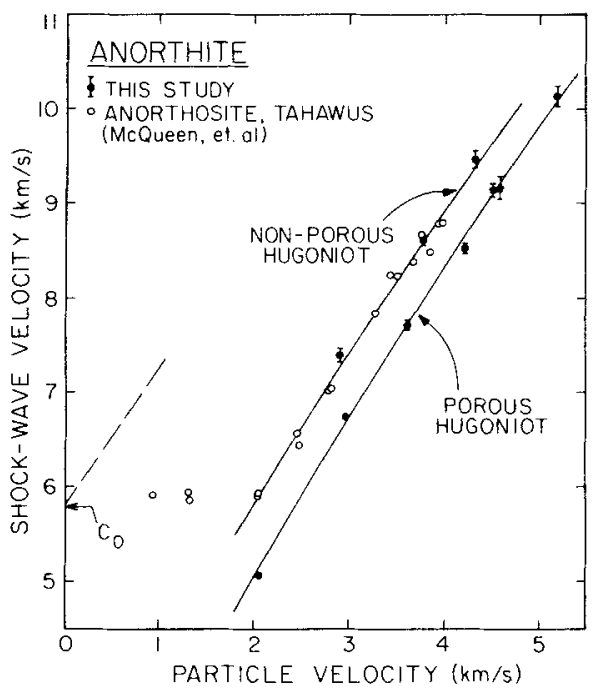

Figure 2. The data and Hugoniot curves from Fig. 1 shown in the shock-wave velocity versus particle velocity plane. The dashed line corresponds to the theoretical Hugoniot of (untransformed) anorthite, while $c_{0}$ shows the bulk sound speed of anorthite from Liebermann \& Ringwood (1976). 
望学

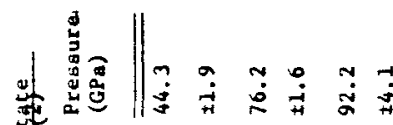

जी

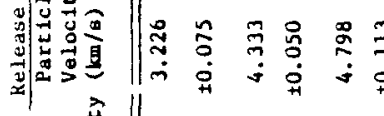

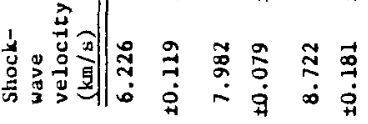

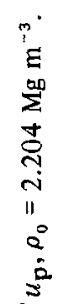

Downloaded from http://gji.oxfordjournalę요

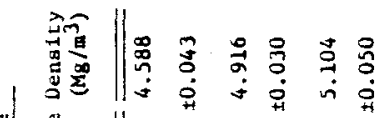

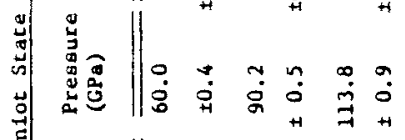

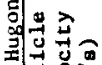

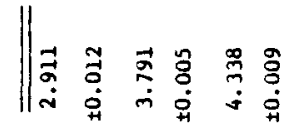

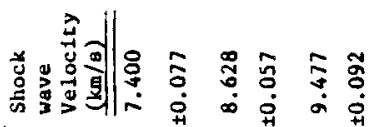

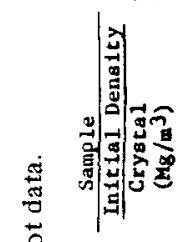

(1)

量

$\widehat{3}$

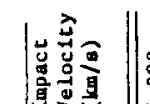

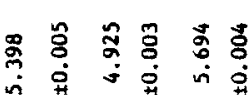

.

+
+
0
-1
-1
11
-1

$\stackrel{\frac{60}{5}}{=}$

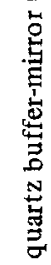

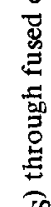

क
D
0
0
0
0
0
0
0
0
0

突

芯导

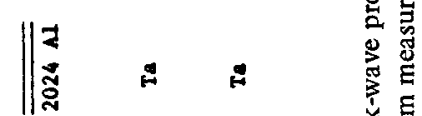

递

方

总方

$\|$ 琞 के

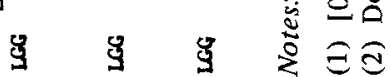




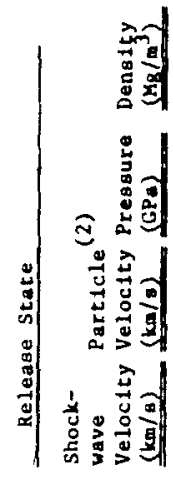

语武 Dow

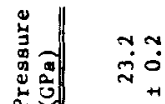

艺㝴

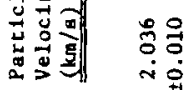

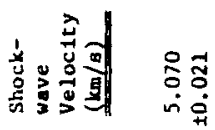

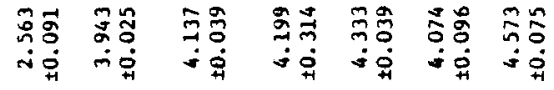

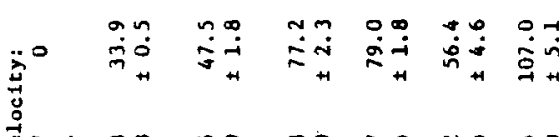

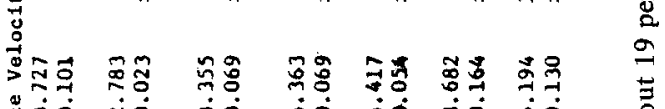

迡斻

岁

乡

ownloaded from http://gji.oxfordjournals.org/ at California Instittute of Technology on October

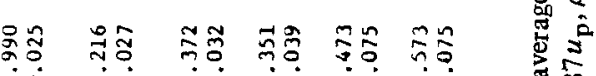

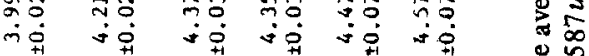

?

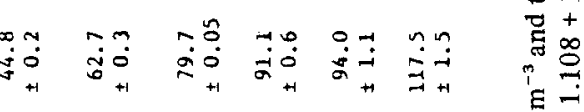

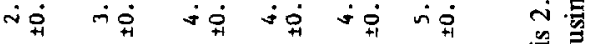

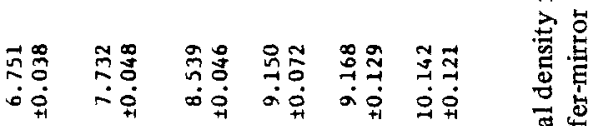

要

它

蛋若

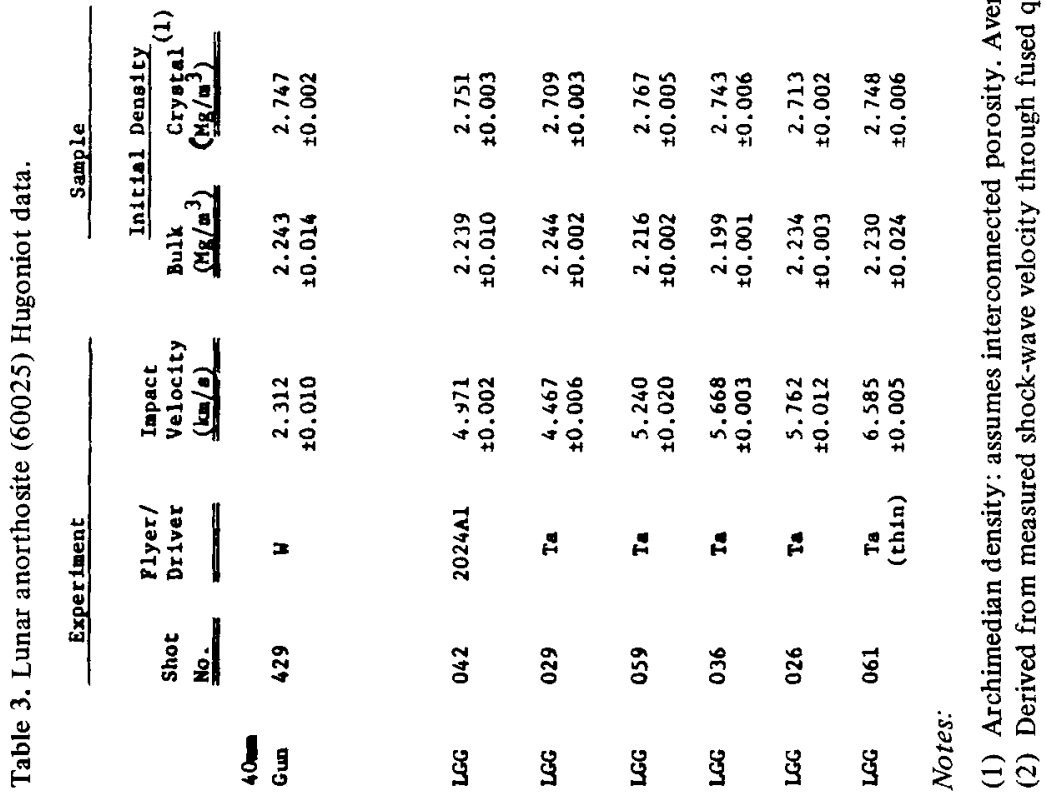


Table 4. Anorthite: Hugoniot equations of state.

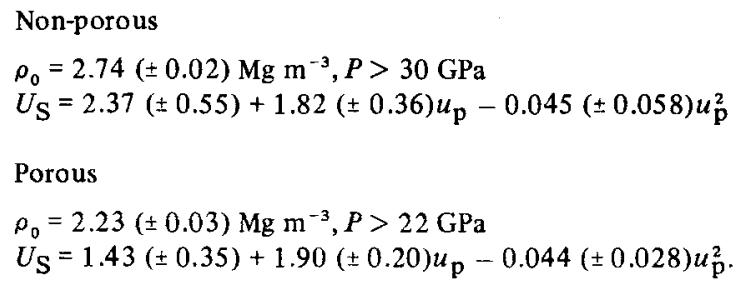

'mixed-phase' region extends from approximately 10.3 to $33.0 \mathrm{GPa}$ for the non-porous samples. The porous and non-porous data in the 'high-pressure phase' regime can be characterized by the least squares fit, quadratic shock-wave velocity $\left(U_{\mathrm{S}}\right)$ versus particle velocity $\left(u_{\mathrm{p}}\right)$ relations given in Table 4 along with the average initial densities $\left(\rho_{0}\right)$. Although the porous data require a quadratic $U_{\mathrm{S}}-u_{\mathrm{p}}$ relation, this is not the case (statistically) for the non-porous data. It is interesting to note, however, that the porous and non-porous data define essentially parallel trends in Fig. 2.

The release paths shown in Fig. 1 are schematic in that they are constrained by only a single measurement in each case, as shown. In using the Riemann integral to derive these data, the release process is assumed to be isentropic, and hence the release paths are expected to have similar (but slightly smaller) slopes in the pressure-density plane as the Hugoniot. Although strictly isentropic release might seem implausible, Jeanloz \& Ahrens (1979) have recently shown that the effects of entropy production (excluding reactions or phase transformations) are not likely to be large enough to influence the release paths. The results for single-crystal anorthite and, at the higher pressures, porous anorthite are consistent with isentropic unloading. These release paths are significantly different from those documented in the 'mixed-phase' region of feldspars in previous studies (Ahrens, Peterson \& Rosenberg 1969; Grady \& Murri 1976), consistent with the present data being in the "highpressure phase' regime of the Hugoniot. It is difficult to understand, however, why several of the release paths for porous anorthite are steeper than the Hugoniot. Finite-strength effects appear not to be responsible and, although several possible explanations can be advanced, these results are enigmatic ( $c f$. Jeanloz \& Ahrens 1978).

\section{Thermal properties}

By combining shock-wave data from samples of different initial densities, thermal properties at high pressures can be derived with virtually no theoretical constraints involved (e.g. Kormer et al. 1962; McQueen et al. 1970; Jeanloz 1979). In the following, thermal properties will be derived based on successively more extensive assumptions or approximations, however, none of the results are strongly dependent on an assumed form of the hightemperature, high-pressure equation of state because each Hugoniot point represents a direct measurement of a pressure $(P)$-volume $(V)$-internal energy $(E)$ state. The most important assumption is that the porous and non-porous data represent identical (or at least very similar) thermodynamic states, except for the temperatures involved; because of the large energies involved, this appears to be a good approximation (see Jeanloz 1979). For example, despite the fact that the porous Hugoniot probably represents molten anorthite to a large extent, the effect of melting on density at a given (high) pressure is small: at zero pressure, a volume change on melting of only 4 per cent is found (Skinner 1966), and at high pressures this undoubtedly decreases to within the few per cent accuracy of the 
Hugoniot data. Hence the effect of melting on the energies or compressibilities involved are not likely to be resolvable, and no anomalies which could be ascribed to melting are seen among the data considered here. Each datum probably represents an average over a heterogeneous thermal state achieved on the Hugoniot, particularly for the porous anorthite in which extremely high temperatures can be achieved along grain boundaries (e.g. Belyakov, Lifshitz \& Rodionov 1974; Belyakov, Rodionov \& Samosadnyi 1977; also, Grady 1977). However, for the hydrostatic condition which is (at least approximately) achieved along the Hugoniot, temperature perturbations result in density (rather than pressure) variations. Thus, temperature variations between $10^{4}$ and $10^{5} \mathrm{~K}$, and concentrated within about 10 per cent of the sample, could readily be concealed within the $1-2$ per cent accuracy to which Hugoniot densities were determined in this study. At these high temperatures other processes, such as radiative thermal conductivity, are likely to become important enough to preclude more extreme temperature variations. The reproducibility of the present data also suggest that an adequate thermal average has been measured.

The porous and non-porous data are directly inverted to yield values of the Grüneisen parameter $\left(\gamma \equiv V(\partial P / \partial E)_{V}\right)$ based on the Hugoniots given in Table 4 . At a given volume $\left(V_{\mathrm{H}}\right)$ the Grüneisen parameter is given by:

$\gamma\left(V_{\mathrm{H}}\right)=V_{\mathrm{H}} \frac{2\left(P_{\mathrm{H}}^{\mathrm{P}}-P_{\mathrm{H}}^{\mathrm{N}}\right)}{P_{\mathrm{H}}^{\mathrm{P}}\left(V_{0}^{\mathrm{N}}-V_{\mathrm{H}}\right)-P_{\mathrm{H}}^{\mathrm{N}}\left(V_{0}^{\mathrm{P}}-V_{\mathrm{H}}\right)}$,

where 0 and $H$ subscripts represent zero-pressure and Hugoniot states, while $\mathrm{P}$ and $\mathrm{N}$ indices refer to porous and non-porous samples respectively (further details are given in Jeanloz 1979). Equation (1) yields values of $\gamma$ which are averaged over large temperature intervals and it is assumed here, as is commonly done (e.g. McQueen et al. 1970; Wallace 1972), that $\gamma$ is essentially independent of temperature. Independent analyses suggest this to commonly be the case (e.g. Anderson et al. 1968; Jeanloz 1979), and the porous data for anorthite, although available within only a small variation of initial porosities, are also consistent with a temperature-independent Grüneisen parameter.

The resulting values of $\gamma$ are shown in Fig. 3 in which each datum point represents the offset of a porous Hugoniot point from the non-porous Hugoniot. The error bars largely reflect uncertainties in the non-porous Hugoniot which must be extrapolated downward considerably in pressure (or density) for this analysis. Because the porous Hugoniot is quite

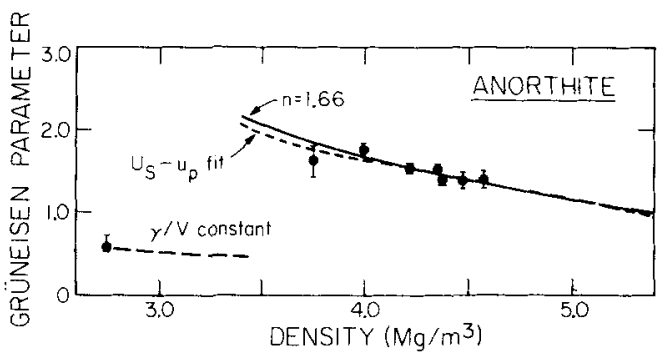

Figure 3. Grüneisen parameter of anorthite as a function of density. The present data at densities above $3.5 \mathrm{Mg} \mathrm{m}^{-3}$ are shown as individual points which can be fit by a power law in density with an exponent of 1.66 (equation 2). Alternatively, the best-fit Hugoniots of Table 4 imply values of the Grüneisen parameter which are given by the short-dashed curve. The zero-pressure value for anorthite is shown at a density of $2.74 \mathrm{Mg} \mathrm{m}^{-3}$ along with an assumed volume dependence (long dashes). Data sources: Liebermann \& Ringwood (1976); Rigby \& Green, and Floyd quoted in Touloukian et al. (1977); Robie et al. (1978). 
well constrained by our data, a smooth $\gamma(\rho)$ curve can be derived from the parameters listed in Table 4 (Fig. 3) which compares favourably with a power-law fit to the data

$$
\gamma=2.20(3.40 \mathrm{~V})^{1.66( \pm 0.42)}
$$

with $V$ in $\mathrm{m}^{3} \mathrm{Mg}^{-1}$ (based on $\rho_{0}=3.40 \mathrm{Mg} \mathrm{m}^{-3}$ : see below). It is interesting to note that the exponent in equation (2) is larger than 1, as in the case of iron (Jeanloz 1979), although a value of unity is often assumed for the volume dependence of the Grüneisen parameter (e.g. McQueen et al. 1967, 1970; Brennan \& Stacey 1979).

The most striking feature in Fig. 3 is the dramatic increase in $\gamma$ from its zero-pressure value, for the high density states achieved along the anorthite Hugoniot. The Grüneisen parameter is expected to decrease upon compression (as shown by the present data), hence the increase shown in Fig. 3 at $\rho<3.7 \mathrm{Mg} \mathrm{m}^{-3}$ directly reflects the large increase in zerothorder anharmonic (quasi-harmonic) contributions to the thermal properties of anorthite as it is compressed through the 'mixed-phase' region. It may seem surprising that anharmonic properties increase across such a density jump, however, this effect is seen in other cases, such as the phase transitions in Fe, Bi and halides (Jeanloz 1979; Ramakrishnan, Hardy \& Kennedy 1979). This is consistent with the standard interpretation of the 'mixed-phase' region of the Hugoniot (e.g. McQueen et al. 1967) as representing a polymorphic transformation to a high-density, high-pressure phase, perhaps with increased cation-coordination number. In general, an increase in coordination number is accompanied by an increase in average bond length and hence an increase in anharmonicity might be expected. However, other effects are also available to explain the observed increase in $\gamma$ with density, such as slight changes in the nature of the interatomic bonding (e.g. Megaw 1938). In any case, an increase in anharmonic contributions to the thermodynamic properties of anorthite when shock-compressed into its high-density state is clearly indicated by the present data.

Given a knowledge of the Grüneisen parameter, an isentropic bulk modulus $\left(K_{\mathrm{S}}\right)$ or bulk sound speed $(c)$ can be determined from the slope of the Hugoniot at any given density (or pressure) by means of the following equation (e.g. Al'tschuler et al. 1960; McQueen et al. $1967,1970)$

$\left.\rho_{\mathrm{H}} c_{\mathrm{H}}^{2}=K_{\mathrm{S}}\right)_{\mathrm{H}}=\left(\frac{d P_{\mathrm{H}}}{d V_{\mathrm{H}}}\right)\left[\left(V_{0}^{\mathrm{P}}-V_{\mathrm{H}}\right) \frac{\gamma}{2 V_{\mathrm{H}}}-1\right] V_{\mathrm{H}}+1 / 2 P_{\mathrm{H}}^{\mathrm{N}} \gamma$

This equation is derived by assuming a Mie-Grüneisen form for the equation of state, however, the thermal correction to the Hugoniot slope is small and is experimentally constrained (through $\gamma$ ), therefore minimizing the effect of this assumption. The resulting values of $K_{\mathrm{S}}$ along the Hugoniot of anorthite are shown in Fig. 4. Again, the individual points are determined by the offset of the porous anorthite data while the curves for $K_{\mathrm{S}}$ along the porous and non-porous Hugoniots are determined from the parameters given in Table 4 (cf. Jeanloz 1979). The error bars on the data mainly reflect uncertainties in the fit to the non-porous Hugoniot, particularly of its slope. Significantly, both the porous and nonporous Hugoniots are well enough constrained to directly yield measurements of the bulk modulus of anorthite at high pressures and at widely varying temperatures (corresponding to different initial porosity). However, in order to quantitatively separate the thermal and compressional effects on the bulk modulus, a more complete model must be derived for anorthite under shock conditions, as is done below. Such a model yields estimates of the temperatures along the porous and non-porous Hugoniots, thus allowing the isotherms shown in Fig. 4 to be determined.

Sound velocities have been independently measured in (alkali) feldspars under shock by means of unloading waves (Grady, Murri \& DeCarli 1975). These velocities were considered 


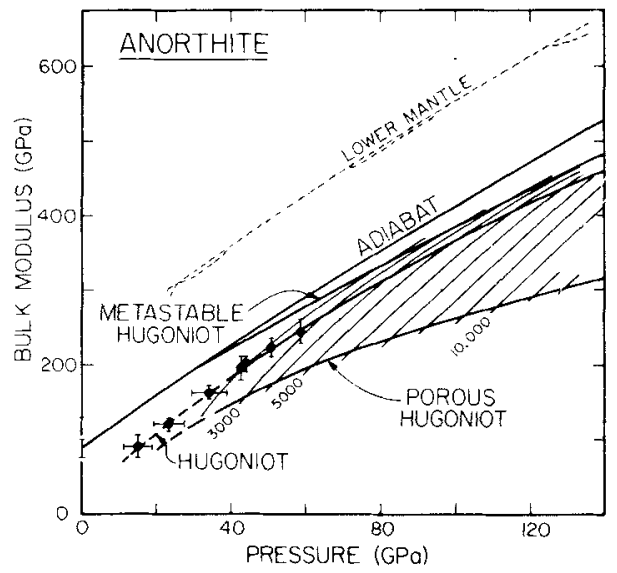

Figure 4. Adiabatic bulk modulus of anorthite as a function of pressure and temperature based on the present shock-wave data, compared with seismological values for the lower mantle (Dziewonski, Hales \& Lapwood 1975; Anderson \& Hart 1976). Bulk moduli based directly on data are shown for the (nonporous) Hugoniot and porous Hugoniot, along with values along the adiabat and metastable Hugoniot derived from the reduced Hugoniot data. Isotherms based on the calculated temperatures are also given (thin, solid lines), with temperatures given in $\mathrm{K}$.

to be anomalously low, and hence were interpreted as being bulk (rather than longitudinal) velocities, suggesting the possibility of partial melting during shock loading. The present results, however, yield lower bulk sound speeds along the Hugoniot than were expected by Grady and co-workers, thus precluding the need to invoke their 'shear-band' melting. In fact, a direct comparison of their velocities with the present data allows the shear modulus along the Hugoniot of feldspar to be calculated. This yields $\mu \sim 117.3$ and $118.3 \mathrm{GPa}$ (Poisson's ratio of $0.21-0.26$ ) at pressures of 34.5 and $46.0 \mathrm{GPa}$ respectively, assuming that Grady et al. measured longitudinal velocities. These values for the shear modulus are not unreasonable, but they are only approximate since there are significant uncertainties in the data sets which are being compared and compositional differences between the samples are ignored. In any case, this analysis suggests that non-porous feldspar melts along the Hugoniot only at pressures significantly higher than $46 \mathrm{GPa}$. Similar comparisons of unloading wave velocities with independently determined bulk compressibilities have been used to determine the shear properties of metals to pressures well in excess of $100 \mathrm{GPa}$ (e.g. Al'tschuler, Brazhnik \& Telegin 1971; Simonov \& Chekin 1975).

Anorthite behaves as though it undergoes a major phase transformation under shock, as exemplified by the large density increase and anomalously high (apparent) compressibility through the 'mixed-phase' region. Without specifying the details of such a transformation, the important consequence is that the properties of anorthite along the 'high-pressure phase' branch of the Hugoniot must be referenced to a zero-pressure density which is significantly higher than the initial density of anorthite and that the densification through the 'mixedphase' region is reflected not as a thermal energy but as a potential energy which is imparted to the (static) lattice: that is, an energy of transformation $\left(\Delta E_{\mathrm{tr}}\right)$. By comparing the theoretical (untransformed) Hugoniot with the non-porous Hugoniot of anorthite at about $30( \pm 10) \mathrm{GPa}$ pressure, a volume decrease of about 20 per cent is found associated with the 'mixed-phase' region. Hence, the high-pressure anorthite data correspond to a state with a zero-pressure density $\rho_{02}=3.40( \pm 0.1) \mathrm{Mg} \mathrm{m}^{-3}$, a Grüneisen parameter given by equation (2) and with $\Delta E_{\mathrm{tr}} \sim 200 \pm 60 \mathrm{~kJ} \mathrm{~mol}^{-1}$. Here, $\Delta S_{\mathrm{tr}} \sim 0$ was assumed and the transition pressure of $10.3 \mathrm{GPa}$ derived above was used. 
With these parameters constrained, a principal adiabat (or isentrope) can immediately be derived for the porous and non-porous anorthite data corresponding to the "high-pressure phase' branch of the Hugoniot. The approach used here was to find a least-squares fit to the data with the adiabat given by either third- or fourth-order, Eulerian finite-strain theory. The appropriate equations for the forward problem (e.g. McQueen et al. 1963; Davies 1973) are readily converted to normal equations of the form:

$$
\frac{2}{3}\left\{\left[1-\frac{\gamma}{2}\left(\frac{\rho_{\mathrm{H}}}{\rho_{01}}-1\right)\right] P_{\mathrm{H}}+\gamma \rho_{\mathrm{H}} \Delta E_{\mathrm{tr}}\right\}\left\{\chi^{3}\left(\chi^{2}-1\right) \xi\right\}^{-1}=\beta_{0}+\beta_{1}\left(\chi^{2}-1\right)\left[\frac{\gamma}{2}\left(\chi^{2}-1\right)-\chi^{2}\right] \zeta^{-1}
$$

with

$$
\begin{aligned}
& \zeta=\chi^{2}-\frac{3}{4} \gamma\left(\chi^{2}-1\right)+\xi_{2}\left(\chi^{2}-1\right)^{2}\left[\chi^{2}-\frac{3}{8} \gamma\left(\chi^{2}-1\right)\right] \\
& \xi_{2}=\frac{3}{8} K_{02} K_{02}^{\prime \prime}+\frac{3}{8} K_{02}^{\prime}\left(K_{02}^{\prime}-7\right)+\frac{143}{24} \\
& \chi=\left(\rho / \rho_{02}\right)^{1 / 3} .
\end{aligned}
$$

The least-squares solution to equation (4) yields best estimates of the parameters $\beta_{0}=K_{02}$ and $\beta_{1}=K_{02} \xi_{1}$, where $\xi_{1}=3 / 4\left(4-K_{02}^{\prime}\right)$ and all properties refer to the adiabat centred at $\rho_{02}$ (primes indicate pressure derivatives). Although equation (4) can easily be extended to the multivariate case in which $\xi_{2}$ and $\Delta E_{\mathrm{tr}}$ could be independently estimated, these variables are so poorly constrained by a fit to the data (at least in the present case) that no further information is gained. A third-order fit (corresponding to a Birch-Murnaghan adiabat) is derived by setting $\xi_{2}=0$ in equation ( $5 \mathrm{a}$ ), resulting in

$K_{0 \mathrm{~S}}=86.5( \pm 10) \mathrm{GPa}$

$K_{0 S}^{\prime}=3.93( \pm 0.20)$

$K_{0 \mathrm{~S}} K_{0 \mathrm{~S}}^{\prime \prime}=-3.45( \pm 0.35)$

for the adiabat corresponding to the 'high-pressure phase' Hugoniot of anorthite; the value in equation (6c) comes from equation (5b). Alternatively, fourth-order solutions can be found for equation (4), however, these do not improve the fit to the data significantly and do not change the resulting adiabat markedly. For example, letting $K_{0 \mathrm{~S}}^{\prime \prime}=0\left(\xi_{2}=1.49\right)$ yields $K_{0 \mathrm{~S}}=95.4 \mathrm{GPa}, K_{0 \mathrm{~S}}^{\prime}=2.93$ and an adiabat which is within about 0.7 per cent in density from that determined by equation (6). It is interesting that $K_{0}^{\prime}$ is essentially equal to 4 (equation $6 \mathrm{~b}$ ), the value for the Birch, second order equation of state, whereas neither the Slater, Dugdale-MacDonald nor Free Volume estimates of $\gamma$ based on this value agree with the present data (e.g. Zharkov \& Kalinin 1971).

The bulk modulus given in equation (6a) is surprisingly low given the large increase in density from $\rho_{01}=2.74 \mathrm{Mg} \mathrm{m}^{-3}$ to $\rho_{02}=3.4 \mathrm{Mg} \mathrm{m}^{-3}$ (compare with $K_{0 \mathrm{~S}}=92 \mathrm{GPa}$ for anorthite at zero pressure: Liebermann \& Ringwood 1976), however, this conclusion is in complete agreement with the results originally derived by McQueen et al. (1967). Because the Grüneisen parameter was not independently known in that earlier study, they derived solutions for the principal adiabat of the 'high-pressure phase' Hugoniot of anorthosite using both low $\left(\gamma_{0}=1.13\right)$ and high $\left(\gamma_{0}=1.73\right)$ values of $\gamma$ (they assumed $\gamma / V=$ constant $)$. The 
values of $\gamma$ measured in the present study clearly favour the latter solution, which resulted in $\rho_{02}=3.46 \mathrm{Mg} \mathrm{m}^{-3}, K_{0 \mathrm{~S}}=88 \mathrm{GPa}$ and $K_{\mathrm{OS}}^{\prime}=3.93$, in excellent agreement with the values found here despite the different formalism used to reduce the Hugoniot data. Ironically, subsequent work in which the seismic equation of state of Anderson $(1967,1969)$ was assumed to hold (Anderson \& Kanamori 1968; Ahrens, Anderson \& Ringwood 1969; Davies \& Anderson 1971) had tended to favour the low- $\gamma$ solution of McQueen and coworkers $\left(\rho_{02}=3.53 \mathrm{Mg} \mathrm{m}^{-3}, K_{0 \mathrm{~S}}=112 \mathrm{GPa}\right)$, resulting in values of $\rho_{0_{2}}$ between 3.57 and $3.71 \mathrm{Mg} \mathrm{m}^{-3}$. In the present study, no solution could be found using equations (2) and (4) which fit the data and which was consistent with either form of the seismic equation of state (Anderson 1967, 1969); it is worth noting that the seismic parameter and density of anorthite are not consistent with the seismic equation of state at zero pressure.

The present results (using equations 6) are shown in Fig. 5, along with the non-porous and porous Hugoniots directly determined by the data. A metastable Hugoniot for anorthite (centred on $\rho_{02}=3.4 \mathrm{Mg} \mathrm{m}^{-3}$ ) can be derived by rearranging equation (4), with $\rho_{01}=\rho_{02}$ and $\Delta E_{\mathrm{tr}}=0$. It is shown in Fig. 5 along with a Hugoniot calculated for 10 per cent initially porous anorthite, which is derived from equations (1) and (2), as can be done for Hugoniots corresponding to arbitrary porosities. The bulk moduli along the adiabat, metastable Hugoniot and calculated porous Hugoniot(s) then follow directly from the theory of finite strains (Birch 1938, 1947; Davies 1973) or, for the Hugoniots, from equation (3); the results are displayed in Fig. 4 (the calculated, porous Hugoniot results are left off for clarity).

In order to derive the temperatures along the compression curves shown in Fig. 5, a model for the specific heat at constant volume $\left(C_{\mathrm{v}}\right)$ must be constructed, except for the case of the adiabat along which the temperature is completely determined by the Grüneisen parameter:

$T_{\mathrm{s}}(\rho)=T_{0 \mathrm{~S}} \exp \left(\frac{\gamma_{0}-\gamma}{n}\right)$

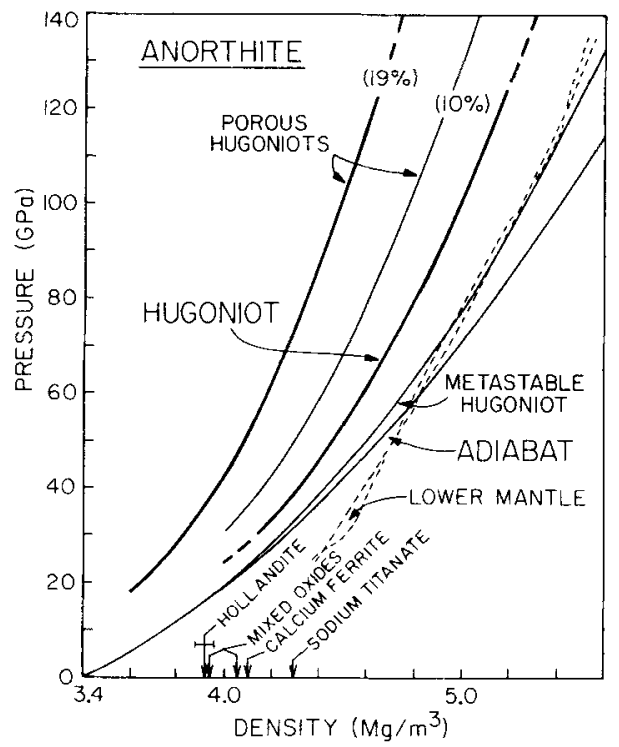

Figure 5. Hugoniots given by the data on anorthite (heavy lines) are compared with the derived compression curves based on the present reduction of the shock-wave data (thin lines). The density in the lower mantle according to Dziewonski et al. (1975) and Anderson \& Hart (1976) is presented (dashed lines), as are the zero-pressure densities of candidate high-pressure assemblages corresponding to anorthite (see Table 5). 
where $\gamma(\rho)$ is given by equation (2): $\gamma_{0}=2.20, n=1.66$ and $T_{0 \mathrm{~S}}=300 \mathrm{~K}$. The temperature along any compression curve (e.g. a Hugoniot) can be calculated from:

$T(P, \rho)=T_{\mathrm{s}}(\rho)+\int_{P_{\mathrm{s}}}^{P} \frac{V d P^{\prime}}{\gamma C_{\mathrm{v}}} ;$

subscript $\mathrm{s}$ indicates evaluated along the principal adiabat and $P^{\prime}$ is a dummy variable. In order to solve equation (8), a simple Debye-Grüneisen model is used to evaluate the specific heat, as has commonly been done in previous studies (e.g. Ahrens et al. 1969b; McQueen et al. 1970). A (high-temperature) value of the Debye temperature $\theta_{1} \sim 1000 \mathrm{~K}$ was found from the zero-pressure specific heat data of anorthite (Robie, Hemingway \& Fisher 1978), however, this must be renormalized to the high-pressure state centred at $\rho_{02}=3.4 \mathrm{Mg} \mathrm{m}^{-3}$. According to Debye theory, the characteristic temperature is proportional to both a mean sound velocity and density, however, because the mean velocity of the highpressure state is unknown, (and velocity systematics apparently do not satisfy the data) the following relation was used (see Anderson et al. 1968, for example):

$\theta_{2} \sim \theta_{1}\left(\rho_{02} / \rho_{01}\right)^{1 / 3}=1075 \mathrm{~K}$.

In equation (9), the mean velocity is assumed constant in going to the high-pressure state and only the density jump is accounted for. This is a rough approximation, but the approximations used here mainly affect the computed results at temperatures less than $\theta$ : about 1100 to $2200 \mathrm{~K}$ for pressures up to $120 \mathrm{GPa}$. Note that the Debye temperature depends on volume according to a relation analogous to equation (7). Because the temperature along the adiabat is given independently, only the temperature along the metastable Hugoniot below about $80 \mathrm{GPa}$ is likely to be seriously affected either by the use of the Debye-Grüneisen model or by the choice of $\theta_{2}$. We note that higher than zeroth order anharmonic contributions to the specific heat, such as the linear $-T$ term (e.g. Wallace 1972), are ignored although they may alter the specific heat somewhat at high temperatures. The overall effect is not likely to be very significant, however, particularly because the higher order anharmonicity is expected to decrease rapidly upon compression (Zharkov \& Kalinin 1971).

The temperatures calculated in this fashion are shown in Fig. 6. The largest uncertainties arise from uncertainties in $\Delta E_{\mathrm{tr}}$, followed by uncertainties in the principal adiabat of Fig. 5 .

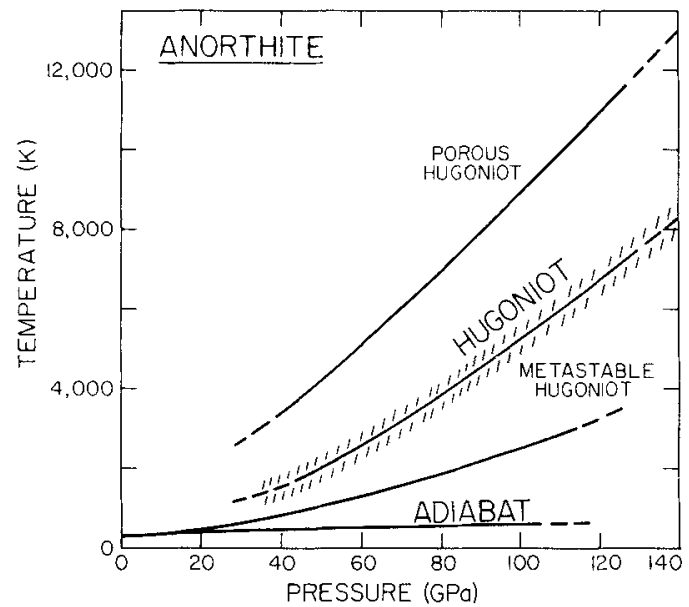

Figure 6. Calculated temperatures along the porous (19 per cent) and non-porous Hugoniot of anorthite, as well as the derived adiabat and metastable Hugoniot. Estimated errors are shown for the Hugoniot temperatures. 
This might be expected since a change in $\Delta E_{\mathrm{tr}}$ from 200 to $300 \mathrm{~kJ} \mathrm{~mol}^{-1}$ involves a temperature change $\delta T \sim \delta E / C_{\mathrm{v}} \sim 100 / 0.32 \sim 300 \mathrm{~K}$ at a given density, and $\Delta E_{\mathrm{tr}}$ is not very well constrained.

The calculated temperatures also allow isotherms to be found for the high-temperature, high-pressure bulk modulus data in Fig. 4. Given the uncertainties and extrapolations involved these isotherms must be considered somewhat schematic, but it is interesting to note the change in pressure derivative of the bulk modulus with temperature and also the relative insensitivity of the bulk modulus to temperature at high pressures and moderately low temperatures. For example at $100 \mathrm{GPa},\left|\left(\partial K_{\mathrm{S}} / \partial T\right)_{\mathrm{P}}\right|$ apparently increases from about $6 \times 10^{-3} \mathrm{GPa} \mathrm{K}^{-1}$ at $4000 \mathrm{~K}$ to about $30 \times 10^{-3} \mathrm{GPa} \mathrm{K}^{-1}$ at $7000 \mathrm{~K}$, a value typical of the region of broadly spaced isotherms between the porous and non-porous Hugoniot curves in Fig. 4. This range of values is also compatible with the low pressure data summarized by Anderson et al. (1968).

The coefficient of thermal expansion is given by

$$
\alpha=\gamma C_{\mathrm{v}}\left(V K_{\mathrm{S}}-\gamma^{2} T C_{\mathrm{v}}\right)^{-1}=\frac{\gamma C_{\mathrm{p}}}{V K_{\mathrm{S}}}
$$

in which the specific heat at constant pressure $\left(C_{\mathrm{p}}\right)$ is distinguished from $C_{\mathrm{v}}$. Equation (10) can be solved as a function of pressure and temperature from the present results on

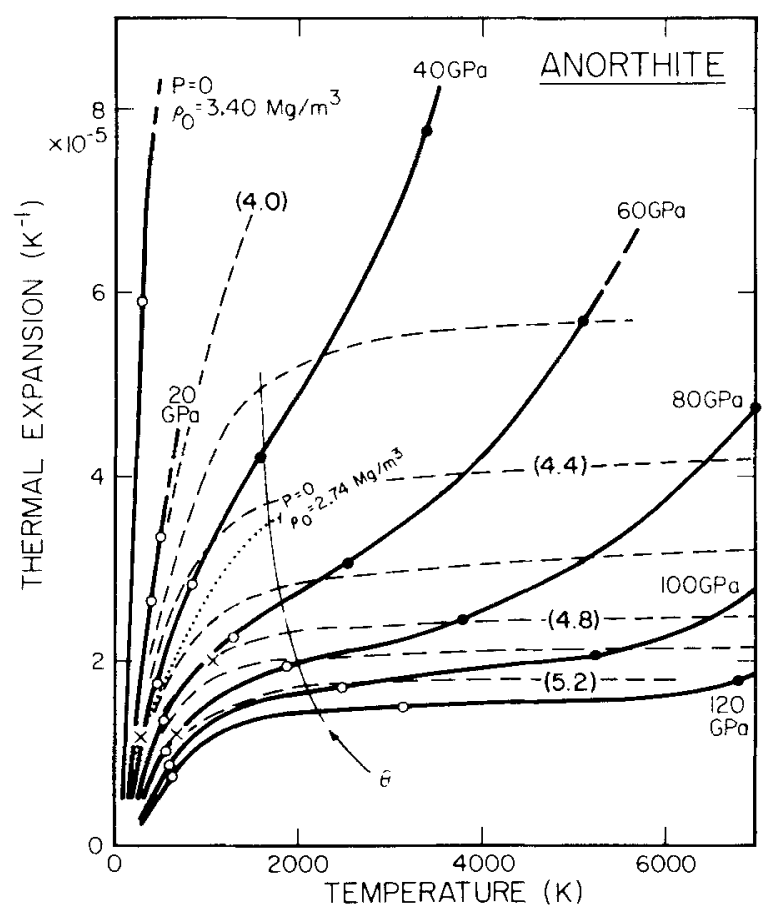

Figure 7. Coefficient of thermal expansion of anorthite as a function of pressure and temperature. Isobars (heavy curves) and density isopleths (thin, dashed curves; densities in $\mathrm{Mg} \mathrm{m}^{-3}$ in parentheses) are shown for the high-pressure state of anorthite. The Debye temperature $(\theta)$ used in this analysis is also given, as a function of density (or pressure). Along each isobar, the circles correspond to intersections with, respectively, the adiabat (open), metastable Hugoniot (open), Hugoniot (filled) and porous Hugoniot (filled) with increasing temperature. For comparison, the thermal expansion of anorthite measured at zeropressure is shown with a dotted curve extending to the melting temperature (marked) according to a fit to the data of Rigby \& Green, and Floyd quoted in Touloukian et al. (1977), and also as crosses from the work of Kozu et al. (quoted in Skinner 1966). 
anorthite, as shown in Fig. 7 in which both isobars and isochores are displayed. For comparison, the zero-pressure data for anorthite and the high-pressure Debye temperature are also shown. At temperatures below $\theta$, the values of $\alpha$ in Fig. 7 are subject to errors due to the possible inadequacies of the specific heat model used. However, the thermal expansion must vanish as the temperature goes to zero and the general features of Fig. 7 are not strongly model-dependent.

Although the zero-pressure thermal expansion of anorthite is subject to some uncertainty, it is significantly lower than the low-pressure thermal expansions derived from the 'highpressure phase' Hugoniot data (Fig. 7). As with the Grüneisen parameter, the thermal expansion of anorthite increases considerably upon compression to the high-pressure state $\left(\rho_{0}=3.40 \mathrm{Mg} \mathrm{m}^{-3}\right)$, whence it decreases with increasing pressure. Again, this can be viewed in terms of anharmonic effects increasing sharply at a pressure-induced phase transformation, whereas pressure decreases both $\gamma$ and $\alpha$ for a given phase. This decrease of thermal expansion with compression can be approximately related to the bulk modulus isotherms in Fig. 4 since

$\left(\frac{\partial \alpha}{\partial P}\right)_{\mathrm{T}}=K_{\mathrm{T}}^{-2}\left(\frac{\partial K_{\mathrm{T}}}{\partial T}\right)_{\mathrm{P}} \sim K_{\mathrm{S}}^{-2}\left(\frac{\partial K_{\mathrm{S}}}{\partial T}\right)_{\mathrm{P}}$

( $K_{\mathrm{T}}$ is the isothermal bulk modulus).

At high temperatures $(T>\theta) \alpha$ increases with temperature, particularly at low pressures. This is due to both zeroth and first-order anharmonic effects which are approximately of equal importance in increasing $\alpha$ : the former through $C_{\mathrm{p}}=(1+\alpha \gamma T) C_{\mathrm{v}}$, while the latter derive from the decrease in the bulk modulus with temperature. It is important to note that these anharmonic contributions decrease more rapidly with pressure than does the zeroorder effect embodied in the thermal expansion itself. Hence at pressures above $80-100$ $\mathrm{GPa}, \alpha$ attains a 'saturated', high-temperature value above the Debye temperature. In this saturated regime, the calculated values of the thermal expansion are virtually modelindependent since the Dulong-Petit value of $C_{\mathrm{v}}$ provides a good approximation for $C_{\mathrm{p}}$ and all other variables in equation (10) are experimentally constrained (cf. Jeanloz 1979). Furthermore, the relative decrease in importance of higher-order anharmonic contributions relative to the lower-order (e.g. strictly quasi-harmonic) effects provides additional justification for the simple Debye-Grüneisen model which was used for $C_{\mathrm{v}}$. From the present data, the second Grüneisen parameter (e.g. Anderson et al. 1968)

$\delta_{\mathrm{S}}=\alpha K_{\mathrm{S}}\left(\frac{\partial K_{\mathrm{S}}}{\partial T}\right)_{\mathrm{P}}$

appears to be relatively independent of temperature at high pressures and temperatures, as expected. Unfortunately, $\delta_{\mathrm{S}}$ is not very well constrained $\left(\delta_{\mathrm{S}} \sim 2.2 \pm 1.2\right)$, however, it can be evaluated over many tens of GPa and thousands of degrees, and its approximate constancy lends further support to the assumed temperature independence of $\gamma$ (Anderson et al. 1968).

\section{Discussion}

Much of the preceding analysis depends on the inference that anorthite undergoes, in some sense, a phase transformation under shock-loading to pressures above about $10.3 \mathrm{GPa}$. Although previous analyses have assumed this to be the case (e.g. McQueen et al. 1967; Anderson \& Kanamori 1968; Ahrens et al. 1969a; Davies \& Anderson 1971), there is considerable doubt that polymorphic transformation occurs under shock in a fashion directly 
Table 5. Candidate high-pressure phases for anorthite $\left(\mathrm{CaAl}_{2} \mathrm{Si}_{2} \mathrm{O}_{8}\right)$.

$$
\begin{aligned}
& \text { Hollandite phase: }{ }^{\mathrm{VIII}} \mathrm{Ca}^{\mathrm{VI}}\left(\mathrm{Al}_{2} \mathrm{Si}_{2}\right) O_{8}, \rho_{0}=3.92 \mathrm{Mg} \mathrm{m}^{-3} \text { (based on Liu 1978a) }
\end{aligned}
$$

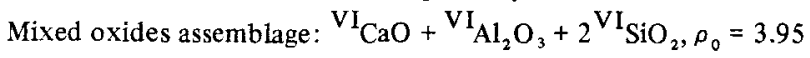

$$
\begin{aligned}
& \text { or } \\
& { }^{\mathrm{VIII}} \mathrm{CaO}+{ }^{\mathrm{VI}} \mathrm{Al}_{2} \mathrm{O}_{3}+2^{\mathrm{VI}} \mathrm{SiO}_{2}, \rho_{0}=4.05 \mathrm{Mg} \mathrm{m}^{-3} \\
& \text { (CaO data from Jeanloz et al. 1979) }
\end{aligned}
$$

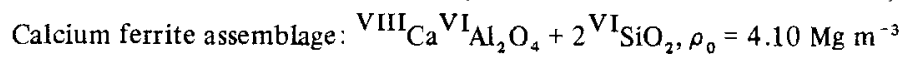

$$
\begin{aligned}
& \text { (Reid \& Ringwood 1969) }
\end{aligned}
$$

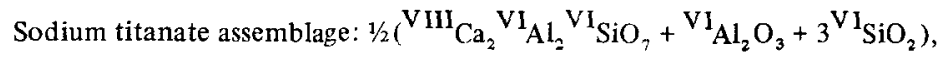

$$
\begin{aligned}
& \rho_{0}=4.29 \mathrm{Mg} \mathrm{m}^{-3} \text { (Liu 1978b; Andersson \& Wadsley 1961) }
\end{aligned}
$$

General references: Ringwood (1975); Robie et al. (1978).

analogous to the phase transformations achieved under static conditions. Because of kinetic limitations, it is quite likely that highly non-equilibrium states are measured in these shock experiments.

One indication of such difficulties arises from a comparison of the present reduction of the Hugoniot data for anorthite in the high-pressure phase' regime with the densities of predicted high-pressure phases or assemblages given in Table 5. As shown in Fig. 5, the zeropressure densities of these candidate high-pressure phases are considerably higher than is found from the reduced Hugoniot data. Although neither the hollandite phase nor the mixed-oxide assemblage have been documented for anorthite, both the calcium ferrite- and sodium titanate-bearing assemblages have been synthesized in calcium-aluminium-silicate systems (Reid \& Ringwood 1969; Liu 1978b). Liu (1978a) has found Na-plagioclase in the hollandite structure, but he has not been able to quench a high-pressure phase of anorthite which occurs above $22 \mathrm{GPa}$ (Liu 1978b). Previous reductions of the Hugoniot data had allowed higher zero-pressure densities than are found here. However, the new data on the Grüneisen parameter preclude such solutions and, as discussed above, the use of the seismic equation of state in those studies appears to be unwarranted. Regardless of other assumptions, no solutions could be found to equations (2), (4) and (5) which fit the data with $\rho_{02}$ larger than about $3.80( \pm 0.10) \mathrm{Mg} \mathrm{m}^{-3}$. In fact, the best fits to the present data resulted from allowing $\rho_{02}<3.0 \mathrm{Mg} \mathrm{m}^{-3}$ and $\Delta E_{\mathrm{tr}}=0$, however, yielding seemingly unphysical values of $K_{0 S}$ (typically less than $50 \mathrm{GPa}$ ). These results suggests that anorthite along the Hugoniot is not transformed to an equilibrium high-pressure polymorph, particularly since less than $30 \mathrm{GPa}$ pressure has been required to find the assemblages listed in Table 5 . A recent study of Jeanloz (1980) finds little evidence for the transformation of (non-porous) silicates in Hugoniot experiments either from observations on shocked olivine samples or from theoretical considerations, and anorthite may provide the first clear case of a silicate achieving significantly more efficient packing under static as compared to dynamic loading.

On the other hand, it is not certain that the results of static high-pressure experiments can be so directly compared with the shock-wave data since the temperatures achieved in the former $(\sim 1000-1500 \mathrm{~K})$ are for the most part considerably less than the temperatures achieved along the Hugoniot. More important though, is the fact that strict equilibrium on a microstructural state need not be required for the kind of analysis presented here. As illustrated above for the case of melting, large energy differences are involved in this analysis such that rather substantial deviations from equilibrium values are not likely to be noted in the Hugoniot data. Furthermore, the evidence for a density increase of about 20 per cent 
above $10 \mathrm{GPa}$, as well as the release adiabats of non-porous anorthite, are all indicative of the behaviour of a high-pressure phase. As discussed by Jeanloz (1980), despite the Hugoniot possibly not representing equilibrium states, the properties measured under shock appear to be very close to their equilibrium values.

There are two problems which must be specifically addressed in this context. Although values of total energy are reasonably well constrained in this analysis, the partitioning of energy may not be. In particular, the amount of thermal energy present, and hence the calculated temperatures, depend on the estimated value of $\Delta E_{\mathrm{tr}}$ : if anorthite undergoes no manner of phase transformation under shock $\Delta E_{\mathrm{tr}}=0$. Jeanloz (1980) used this fact and measured Hugoniot temperatures to argue that some form of (non-equilibrium) transformation apparently occurs in silicates under shock. Similarly, if melting occurs in the anorthite samples this would not affect the bulk thermal or compressional properties severely, but it could change the calculated temperatures dramatically, lowering them (at a given pressure) by possibly $10^{3} \mathrm{~K}$. Since the zero-pressure melting temperature of anorthite is $1830 \mathrm{~K}$, a simple scaling argument suggests that melting may occur at about $70-90 \mathrm{GPa}$ on the nonporous Hugoniot or about $40-60 \mathrm{GPa}$ along the porous Hugoniot. This ambiguity can, however, be directly resolved by way of shock-temperature measurements or by determinations of the shear modulus along the Hugoniot. Higher order effects associated with melting, such as discussed by Grover (1971), are not likely to change the values of properties calculated here by more than $15-20$ per cent even at the highest temperatures shown in Fig. 6; given the important decrease in higher-order anharmonicities with pressure this is likely to be an overly conservative estimate.

The second question is whether the phase(s) along the 'high-pressure phase' Hugoniot evolve continuously with increasing pressure, as might particularly be expected if these are in highly non-equilibrium states. This is equivalent to considering all of the data presented here as being in a 'mixed-phase' regime despite the break in the $U_{\mathrm{S}}-u_{\mathrm{p}}$ relation documented in Fig. 2 ( $c f$. McQueen et al. 1967, for example). Some question has, in fact, been raised for other silicates (Jeanloz \& Ahrens 1977; Jackson \& Ahrens 1979) about stability in the 'high-pressure phase' regime: some evidence suggests continued reaction or evolution along the Hugoniot to pressures of $100 \mathrm{GPa}$ or higher. Although the measured Hugoniot densities might still be used in such a case, the reduction of the Hugoniot data and especially the values of bulk moduli derived above would be meaningless. At present there is no compelling evidence for such a conclusion and counter-examples can be found; however, the inability to independently verify this is problematical. Hence, just as the porous and non-porous Hugoniots are assumed to be directly comparable, the 'high-pressure phase' branch of the Hugoniot is assumed to represent a single (possibly non-equilibrium) phase or assemblage.

\section{Lower mantle}

Since theories involving inhomogeneous accretion of the planets suggest that relatively refractory compounds, such as anorthite, may exist deep within the Earth, it is of interest to compare the present data with seismologically-based models of the lower mantle. In Figs 4 and 5 the density and bulk modulus of the mantle are compared with our data for anorthite. The density of anorthite is compatible with mantle densities, but only for pressures in excess of about 80 to $100 \mathrm{GPa}$. As is evident from Fig. 4, however, the bulk moduli derived here for anorthite at high pressures virtually preclude its being a major component throughout the bulk of the lower mantle ( $c f$. McQueen et al. 1967). If the assumptions made in calculating the bulk moduli are in error, then higher (more compatible) values would be found for anorthite. 
Whereas a recent study on $\mathrm{CaO}$ (Jeanloz et al. 1979) indicates that the lower mantle could be considerably enriched in calcium, the present data do not support anorthite as being a particularly important Ca-bearing mineral in the mantle except possibly in its lowermost portions $\left(D^{\prime \prime}\right.$ region). Because the $D^{\prime \prime}$ region is seismologically anomalous it is not possible to determine its properties with great confidence. Indeed, the evidence for a decrease in velocity gradient through the $\mathrm{D}^{\prime \prime}$ region (see the review of Cleary (1974), for example) is compatible with its containing 10-20 per cent anorthite (or similar refractory compound) according to the present data, while for plausible mantle temperatures (e.g. Stacey 1977; Jeanloz \& Richter 1979) anorthite has a density within about 1 per cent of the observed mantle densities at this level. Although not required, an enrichment of anorthite in the $D^{\prime \prime}$ region is therefore acceptable and would appear to be buoyantly stable. This is consistent with a simple thermal model for the lower mantle presented by Jeanloz \& Richter (1979), in which the $D^{\prime \prime}$ region is chemically distinct from the overlying mantle. An increase in refractory components toward the base of the mantle is in qualitative agreement with inhomogeneous accretion models, however, a simple zone-refining process could produce the same effect in an originally homogeneous mantle heated from below.

\section{Conclusions}

New shock-wave data have been presented for anorthite from which a full high-temperature, high-pressure equation of state has been derived. Whereas anorthite has relatively low values of thermal expansion and Grüneisen parameter at zero pressure, these attain relatively high values in the high density state corresponding to the 'high-pressure phase' Hugoniot, but decrease upon compression as expected. Higher order anharmonic contributions appear to decrease more rapidly with pressure and the thermal expansion therefore saturates to a high temperature value at pressures above about $100 \mathrm{GPa}$. Reduction of the Hugoniot data allows shock temperatures to be calculated and also yields a principal adiabat for the high pressure branch of the Hugoniot. This adiabat has an initial bulk modulus (about $87 \mathrm{GPa}$ ) which is essentially identical to that of anorthite, whereas the initial density is about $3.40 \mathrm{Mg} \mathrm{m}^{-3}$. Because candidate, high-pressure assemblages with significantly higher densities are known for anorthite $\left(4.05-4.30 \mathrm{Mg} \mathrm{m}^{-3}\right)$ and because Hugoniot states are not likely to be at equilibrium, an ambiguity arises in the interpretation of the present data which are assumed to reflect equilibrium properties. Nevertheless, this assumption appears to be valid and no inconsistencies are evident. Significantly, no solutions could be found in reducing the data with zero-pressure densities for the principal adiabat above $3.8-3.9 \mathrm{Mg} \mathrm{m}^{-3}$, or with bulk moduli satisfying the seismic equation of state. A reduction of these data to lower mantle conditions demonstrate that anorthite may be present in amounts of 10-20 per cent in the $D^{\prime \prime}$ region, but it is not likely to be a significant phase elsewhere in the lower mantle because of its apparently small bulk modulus.

\section{Acknowledgments}

We thank Dr Y. Syono for providing anorthite crystals and appreciate the helpful comments of D. E. Grady, L.-G. Liu and the anonymous reviewers. We are also grateful to E. Gelle, J. Long and R. Smith for their help in carrying out the experiments. Work supported by NASA Grant NSG9019. Contribution number 3275, Division of Geological and Planetary Sciences, California Institute of Technology, Pasadena, CA 91125. 


\section{References}

Ahrens, T. J., Anderson, D. L. \& Ringwood, A. E., 1969. Equations of state and crystal structures of high-pressure phases of shocked silicates and oxides, Rev. Geophys., 7, 667-707.

Ahrens, T. J., Petersen, C. F. \& Rosenberg, J. T., 1969. Shock compression of feldspars, J. geophys. Res., 79, 2727-2746.

Ahrens, T. J., Jackson, I. \& Jeanloz, R., 1977. Shock compression and adiabatic release of a titaniferous mare-basalt, Proc. 8th Lunar Sci. Conf., 3437-3455.

Al'tshuler, L. V., Brazhnik, M. I. \& Telegin, G. S., 1971. Strength and elasticity of iron and copper at high shock-wave compression pressures, J. appl. Mech. Tech. Phys., 12, 921-926.

Al'tshuler, L. V., Kormer, S. B., Brazhnik, M. I., Vladimirov, L. A., Speranskaya, M. P. \& Funtikov, A. I., 1960. The isentropic compressibility of aluminium, copper, lead and iron at high pressures, Soviet Phys. JETP, 11, 766-775.

Anderson, D. L., 1967. A seismic equation of state, Geophys. J. R. astr. Soc., 13, 9-30.

Anderson, D. L., 1969. Bulk modulus-density systematics, J. geophys. Res., 74, 3857-3864.

Anderson, D. L., 1975. Chemical plumes in the mantle, Bull. geol. Soc. Am., 86, 1593-1600.

Anderson, D. L. \& Hart, R. S., 1976. An earth model based on free oscillations and body waves, $J$. geophys. Res., 81, 1461-1475.

Anderson, D. L. \& Kanamori, H., 1968. Shock-wave equations of state for tocks and minerals, J. geophys. Res., 73, 6477-6502.

Anderson, O. L., Schreiber, E., Liebermann, R. C. \& Soga, N., 1968. Some elastic constant data on minerals relevant to geophysics, Rev. Geophys., 6, 491-524.

Andersson, S. \& Wadsley, A. D., 1961. The crystal structure of $\mathrm{Na}_{2} \mathrm{Ti}_{3} \mathrm{O}_{7}$, Acta crystall, 14, 1245-1249.

Belyakov, G. V., Lifshitz, L. D. \& Rodionov, V. N., 1974. Shock-wave deformation on an inhomogeneous medium which is modelled by a set of steel balls, Izv. Earth Phys., no. 10,655-667.

Belyakov, G. V., Rodionov, V. N. \& Samosadnyi, V. P., 1977. Heating of porous material under impact compression, Combustion, Explosion Shock Waves, 13, 524-528.

Birch, F., 1938. The effect of pressure upon the elastic parameters of isotropic solids, according to Murnhagan's theory of finite strain, J. appl. Phys., 9, 279-288.

Birch, F., 1947. Finite elastic strain of cubic crystals, Phys. Rev., 71, 809-824.

Btennan, B. J. \& Stacey, F. D., 1979. A thermodynamically based equation of state for the lower mantle, J. geophys. Res., 84, 5535-5539.

Clark, S. P., Turekian, K. K. \& Grossman, L., 1972. Chemical models of the Earth, in The Nature of the Solid Earth, pp. 3-18, ed. Robertson, E. C., McGraw-Hill, New York.

Cleary, J. R., 1974. The D" region, Phys. Earth planet. Interiors, 9, 13-27.

Davies, G. F., 1973. Quasi-harmonic finite strain equations of state of solids, J. Phys. Chem. Solids, 34, $1417-1429$.

Davies, G. F. \& Anderson, D. L., 1971. Revised shock-wave equations of state for high-pressure phases of rocks and minerals, J. geophys. Res., 76, 2617-2627.

Davies, G. F. \& Gaffney, E. S., 1973. Identification of high-pressure phases of rocks and minerals from Hugoniot data, Geophys. J. R. astr. Soc., 33, 165-183.

Dziewonski, A. M., Hales, A. L. \& Lapwood, E. R., 1975. Parametrically simple earth models consistent with geophysical data, Phys. Earth planet. Interiors, 10, 12-48.

Gay, P., 1954. The structure of the plagioclase feldspars V, Miner. Mag., 30, 428-438.

Grady, D. E., 1977. Processes occurring in shock wave compression of rocks and minerals, in HighPressure Research, pp. 389-438, eds Manghnani, M. \& Akimoto, S., Academic Press, New York.

Grady, D. E. \& Murri, W. J., 1976. Dynamic unloading in shock compressed feldspar, Geophys. Res., Lett., 3, 472-474.

Grady, D. E., Murri, W. J. \& DeCarli, P. S., 1975. Hugoniot sound velocities and phase transformations in two silicates, J. geophys. Res., 80, $4857-4861$.

Grossman, L. \& Larimer, J. W., 1974. Early chemical history of the solar system, Rev. Geophys. Space Phys., 12, 71-101.

Grover, R., 1971. Liquid metal equation of state based on scaling, J. Chem. Phys., 55, 3435-3441.

Jackson, I. \& Ahrens, T. J., 1979. Shock-wave compression of single-crystal forsterite, J. geophys. Res., $84,3039-3048$.

Jeanloz, R., 1979. Properties of iron at high pressures and the state of the core, J. geophys. Res., 84, $6059-6069$.

Jeanloz, R., 1980. Shock effects of olivine and implications for Hugoniot data, J. geophys. Res., in press. 
Jeanloz, R. \& Ahrens, T. J., 1977. Pyroxenes and olivines: structural implications of shock-wave data for high pressure phases, in High-Pressure Research, pp. 439-461, eds Manghnani, M. \& Akimoto, S., Academic Press, New York.

Jeanloz, R. \& Ahrens, T. J., 1978. The equation of state of a lunar anorthosite: 60025, Proc. 9th Lunar Planet. Sci. Conf., 2789 2803.

Jeanloz, R. \& Ahrens, T. J., 1979. Release adiabat measurements on minerals: the effect of viscosity, J. geophys. Res., 84, $7545-7548$.

Jeanloz, R. \& Ahrens, T. J., 1980. Equations of state of $\mathrm{FeO}$ and $\mathrm{CaO}$, Geophys. J. R. astr. Soc., 62, $505-528$.

Jeanloz, R., Ahrens, T. J., Mao, H. K. \& Bell, P. M., 1979. B1/B2 transition in CaO from shock-wave and diamond cell experiments, Science, in press.

Jeanloz, R. \& Richter, F. M., 1979. Convection, composition and the thermal state of the lower mantle, J. geophys. Res., 84, $5497-5504$.

Keeler, R. N. \& Royce, E. B., 1971. Shock waves in condensed media, in Physics of High Energy Density, pp. 51-150, eds Caldirola, P. \& Knoepfel, H., Academic Press, New York.

Kormer, S. B., Funtikov, A. I., Urlin, V. D. \& Kolesnikova, A. N., 1962. Dynamic compression of porous metals and the equation of state with variable specific heat at high temperatures, Soviet Phys. $J E T P, 15,477-488$.

Liebermann, R. C. \& Ringwood, A. E., 1976. Elastic properties of anorthite and the nature of the lunar crust, Earth planet. Sci. Lett., 31, 69-79.

Liu, L.-G., 1978a. High-pressure phase transformations of albite, jadeite and nepheline, Earth planet. Sci. Lett., 37, $438-444$.

Liu, L.-G., 1978b. A new high-pressure phase of $\mathrm{Ca}_{2} \mathrm{Al}_{2} \mathrm{SiO}_{7}$ and implications for the earth's interior, Earth planet. Sci. Lett., 40,401-406.

Lyzenga, G. \& Ahrens, T. J., 1978. The relations between the shock-induced free-surface velocity and post-shock density of solids, J. appl. Phys., 49, 201-204.

Marvin, U. B., Wood, J. A. \& Dickey, J. S. Jr, 1970. Ca-Al rich phases in the Allende meteorite, Earth planet. Sci. Lett., 7, 346--350.

McLaren, A. C., 1973. The domain structure of a transitional anorthite: a study by direct latticeresolution electron microscopy, Contrib. Mineral. Petrol., 41, 47-52.

McQueen, R. G., Fritz, J. N. \& Marsh, S. P., 1963. On the equation of state of stishovite, J. geophys. Res., $68,2319-2322$.

McQueen, R. G., Marsh, S. P. \& Fritz, J. N., 1967. Hugoniot equations of state of twelve rocks, $J$. geophys. Res., 72, 4999-5036.

McQueen, R. G., Marsh, S. P., Taylor, J. W., Fritz, J. N. \& Carter, W. J., 1970. The equation of state of solids from shock wave studies, in High Velocity Impact Phenomena, pp. 294-419, ed. Kinslow, R., Academic Press, New York.

Megaw, H. D., 1938. The thermal expansion of crystals in relation to their structure, Z. Kristallog., 100, $58-76$.

Müller, W. F., Wenk, H. R. \& Thomas, G., 1972. Structural variations in anorthite, Contrib. Mineral. Petrol., 34, 304-314.

Ramakrishnan, J., Hardy, R. J. \& Kennedy, G. C., 1979. The Grüneisen parameter of $\mathrm{KBr}, \mathrm{RbCl}$ and Bi through high pressure phase transitions, J. Phys. Chem. Solids, 40, 297-303.

Reid, A. F. \& Ringwood, A. E., 1969. Newly observed high pressure transformations in $\mathrm{Mn}_{3} \mathrm{O}_{4}, \mathrm{CaAl}_{2} \mathrm{O}_{4}$ and $\mathrm{Zr} \mathrm{SiO}$, Earth planet. Sci. Lett., 6, $205-208$.

Rice, M. H., McQueen, R. G. \& Walsh, J. M., 1958. Compressibility of solids by strong shock waves, Solid State Phys., 6, 1-63.

Ringwood, A. E., 1975. Composition and Petrology of the Earth's Mantle, McGraw-Hill, New York, $608 \mathrm{pp}$.

Robie, R. A., Hemingway, B. S. \& Fisher, J. R., 1978. Thermodynamic Properties of Minerals and Related Substances, U.S. Geol. survey Bull, 1452 Washington, D.C., 456 pp.

Simonov, I. V. \& Chekin, B. S., 1975. High-velocity collisions of iron plates, Combustion, Explosion Shock Waves, 11, 237-242.

Skinner, B. J., 1966. Thermal expansion, in Handbook of Physical Constants, ed. Clark, S. P., Geol. Soc. Am. Mem. 97, 75-96.

Stacey, F. D., 1977. A thermal model of the earth, Phys. Earth planet. Interiors, 15, 341-348.

Toulo ukian, Y. S., Kirby, R. K., Taylor, R. E. \& Lee, T. Y. R., 1977. Thermal Expansion, Nonmetallic Solids, IFI Plenum, New York, $1658 \mathrm{pp}$. 
Turekian, K. K. \& Clark, S. P. Jr, 1969. Inhomogeneous accretion model of the earth from the primitive solat nebula, Earth planet. Sci, Lett., 6, 346-348.

Wackerle, J., 1962. Shock-wave compression of quartz, J. appl. Phys., 33, 922-937.

Wallace, D. C., 1972. Thermodynamics of Crystals, J. Wiley \& Sons, New York, 484 pp.

Walsh, J. M. \& Christian, R. H., 1955. Equation of state of metals from shock wave measurements, Phys. Rev., 97, 1544-1556.

Zharkov, V. N. \& Kalinin, V. A., 1971. Equations of State for Solids at High Pressures and Temperatures, Consultants Bureau, New York, 257 pp. 